\title{
Resolution enhancement for ISAR imaging via improved statistical compressive sensing
}

\author{
Lei Zhang ${ }^{1}$, Hongxian Wang ${ }^{1}$ and Zhi-jun Qiao ${ }^{2^{*}}$
}

\begin{abstract}
Developing compressed sensing (CS) theory reveals that optimal reconstruction of an unknown signal can be achieved from very limited observations by utilizing signal sparsity. For inverse synthetic aperture radar (ISAR), the image of an interesting target is generally constructed by limited strong scattering centers, representing strong spatial sparsity. Such prior sparsity intrinsically paves a way to improved ISAR imaging performance. In this paper, we develop a super-resolution algorithm for forming ISAR images from limited observations. When the amplitude of the target scattered field follows an identical Laplace probability distribution, the approach converts super-resolution imaging into sparsity-driven optimization in the Bayesian statistics sense. We show that improved performance is achievable by taking advantage of the meaningful spatial structure of the scattered field. Further, we use the nonidentical Laplace distribution with small scale on strong signal components and large scale on noise to discriminate strong scattering centers from noise. A maximum likelihood estimator combined with a bandwidth extrapolation technique is also developed to estimate the scale parameters. Real measured data processing indicates the proposal can reconstruct the high-resolution image though only limited pulses even with low SNR, which shows advantages over current super-resolution imaging methods.
\end{abstract}

Keywords: Inverse synthetic aperture radar (ISAR), Super-resolution, Compressive sensing (CS), Statistical compressive sensing, Bayesian, Laplace distribution, Nonidentical distribution

\section{Introduction}

High-resolution radar imaging techniques are widely applied in many military and civilian fields, such as target classification and recognition and aircraft traffic control. For success of these applications, sufficient image resolution is required to characterize the scattering and geometric features of the target. Inverse synthetic aperture radar (ISAR) combines the use of pulse compression, flexible pulse repetition frequency (PRF), and target motions (particularly the rotating motion) to generate twodimensional high-resolution imagery. In general, range resolution is determined by the bandwidth of the transmitted signal which is limited by the radar system. To mitigate this limitation, stepped frequency waveforms are usually employed at a price of longer coherent

\footnotetext{
* Correspondence: leizhang@xidian.edu.cn

${ }^{2}$ School of Mathematical and Statistical Sciences, University of Texas-Rio Grande Valley, Edinburg TX 78539, USA

Full list of author information is available at the end of the article
}

processing interval (CPI) [1]. Cross-range resolution is dependent on both the CPI and the target rotational motion from the variation of radar viewing angles. It is well known that CPI should be long enough for high azimuth resolution, which conflicts with modern radar activities such as multi-target tracking and searching [2].

Due to the CPI limitation, achieving high resolution in azimuth with only a few pulses through conventional radar imaging schemes, e.g., range-Doppler (RD) algorithm [3], is difficult. This motivates super-resolution (SR) techniques. SR image formation improves the ability to resolve two closely spaced scatterers in comparison with the Nyquist resolution limit [4], which actually involves uncertainty as the dimension of the image is required to be higher than that of the measurements. Enhancing both the image contrast enrichment and scattering center localization, SR imaging will also reduce scintillation effects associated with unresolved scatterers. A super-resolved image can usually promote the 
probability of correct automatic target recognition (ATR) $[5,6]$. Consequently, a large fraction of the radar imaging research effort has been taken in developing resolution enhancing algorithms. Generally, there are three categories that conventional SR approaches are often sorted into. The first group is the bandwidth extrapolation (BWE) methods. They are based on the fact that the data is analytic in principle and can be fitted into a linear prediction model [2, 7-10]. BWE usually applies some modern spectral estimation techniques to determine the coefficients of the prediction model. Burg's algorithm [2] is one of the most successful estimators. The second general group of SR methods is the parametric spectral estimation techniques. They attempt to estimate parameters of the dominant scattering centers by modern spectral estimation techniques. Probabilistic strategies which model the signal and noise statistically, including maximum a posteriori and maximum entropy $[11,12]$ schemes have also been developed, yielding good performance. Adaptive spectral estimators [13-18] utilize sinusoid parametric model to estimate the coefficients of signals by finding a set of filters adaptively. Among this sort, RELAX [13], [14], which estimates the amplitude and frequency of a multicomponent complex sinusoidal signal by minimizing the residual error energy, is one of the most popular algorithms. Imageries generated by these approaches are inherently free from sidelobes, and resolution relies on the precision of spectral estimation. The third group is nonlinear filtering techniques to reduce side lobes while preserving the width of the main lobe. This group includes space-variant filtering methods such as adaptive sidelobe reduction (ASR) [19] and the related special case of spatially variant apodization (SVA) [20], as well as CLEAN and its modified versions [21, 22]. All the current SR algorithms are more or less sensitive to noise and model error.

Conventional SR imaging can be regarded as a process that recovers a higher dimensional signal from measurements with much fewer degrees of freedom and is deemed to involve uncertainty mathematically. Developing compressive sensing (CS) theory reveals that an unknown sparse signal can be exactly recovered from very limited measurements with high probability by solving a convex $l_{1}$ optimization problem [23-25]. Satisfaction of the restricted isometry property (RIP) allows the uncertainty in the $l_{1}$ optimization problem to be overcome to provide an exact or approximate signal recovery. The essence of CS is to exploit prior knowledge of sparsity of the objective signal. This explains the promising performance of radar imaging via regularization techniques. For ISAR imaging, strong scattering centers exhibiting the scattering organization and geometric configuration of the target usually occupy only a fraction of whole bins in the RD plane. In this sense, the ISAR signal is spatially sparse in RD domain. Such sparsity can be exploited by $l_{1}$ optimization to enhance image quality and feature. Many sparsity-driven SR approaches based on $l_{1}$ optimization have been developed for SAR/ISAR imaging. The feature-enhanced SAR imaging approach $[26,27]$ exploits the sparsity of both image and differential image via the $l_{p}(0<p \leq 1)$ optimization to enhance the point and region feature of the radar image. Particularly, the point-enhanced image formation can extrapolate the data bands beyond the observations, yielding SR imaging. It should be noted that the point-enhanced optimization with $p=1$ is equivalent to the $l_{1}$ optimization of CS. However, the main problem of the feature-enhanced algorithm for radar imaging lies in the difficulty of parameter selection. SR ISAR imaging using the $l_{1}$-FFT, is developed in [28]. Similarly, in [29], a CSbased approach is developed, which can generate a highresolution image with very few pulses, promising promoted performance of ISAR imaging by utilizing spatial sparsity. Based on the assumption that only a few scatterers with different elevations are present in the same range-azimuth resolution cell, CS is exploited in [30,31], to provide a new data acquisition scheme and SR imaging using only a few signal samples for tomographic SAR imagery. In [32], the SR capability and robustness of CS for tomographic SAR imaging have been analyzed in detail. All this work indicates that it is possible to substantially enhance the performance of the radar imaging by exploiting target sparsity. Nevertheless, it is usually not an easy task to characterize this sparsity quantitatively due to the uncertainty and complexity of the real target scattered field, together with the inevitable presence of noise and clutter. This makes some regularization-based methods including the featureenhanced image approach ambiguous in both parameter selection and controlling the sparsity of the recovery.

Generally, it is preferable to represent the sparsity of the target in a statistical manner. In this paper, a SR imaging algorithm is developed wherein the amplitude sparsity of the target scattered field is represented by a Laplace distribution. Based on this, the statistical distribution of the complex-valued scattered field is derived. The SR imaging problem is then converted into solving a sparsity-constraint optimization corresponding to the maximum a posterior (MAP) probability estimation following the Bayesian theory. For simplicity, the proposed algorithm is called Bayesian SR imaging. From Bayesian compressive sensing (BCS) [33] under the identical Laplace distribution assumption, the original Bayesian SR imaging is explicitly coincident with the $l_{1}$-regularization optimization. The sparsity coefficient controls the sparsity of the reconstructed image, which is directly related to the statistics of the target image and noise. We also 
extend the Bayesian SR imaging by using nonidentical Laplace distributions since the scattered field of a target usually follows the energy-assembling and geometric organization of the target. We place Laplace distributions with different scale parameters on each element, by which signal and noise components could be penalized individually in the $l_{1}$ optimization to promote the accuracy of SR imagery reconstruction. A novel scheme to estimate the noise and target statistics parameters are also developed for both the original Bayesian SR and the improved version by combining the constant-falsealarm-ratio (CFAR) [34] and Burg's BWE techniques. To achieve a super-resolved image stably, we propose an iterative procedure where in each stage the aperture data is extrapolated doubly and the maximum likelihood (ML) estimate of the Laplace scale parameter is updated. The Bayesian SR methods are inherently robust to noise and workable with limited pulses. Both simulated and real data experiments are provided to demonstrate the superiority of Bayesian SR imaging methods over the current SR approaches under different circumstances.

The organization of this paper is as follows. Section 2 introduces the Bayesian SR imaging approaches based on identical and nonidentical statistics assumptions. In Section 3, we focus on three issues: statistics estimation, imaging procedure, and a modified Quasi-Newton solver. Finally, in Section 4, simulated and real-data experimental results are given to show the effectiveness of the proposed approaches.

\section{Resolution enhancement with statistical compressive sensing}

\section{A. Signal model}

Considering that an ISAR system observes a maneuvering target and collects a number of pulses coherently, our aim is to achieve a high-resolution image of the target. At first, conventional motion compensation is applied to the raw data followed by range compression and signal alignment to produce the signal $\mathbf{S}$. We assume that the data contains $N$ pulses with index from 0 to $N$ -1 and each pulse composes $M$ range bins. Then, the range-compressed data set is given by $\mathbf{S}=\left[s_{n m}\right]_{N \times M}$. Based on the short CPI assumption, the data can be regarded as a measured patch of the Fourier transform of the target scattered field relevant to some aspect angles and so the echoed signals can be rewritten as

$$
\mathbf{S}=\mathbf{F A}+\boldsymbol{\varepsilon}
$$

where $\mathbf{A}=\left[a_{\bar{n} m}\right]_{\bar{N} \times M}$ is the $\bar{N} \times M(\bar{N}>N)$ superresolved ISAR image whose pixel values correspond to the amplitude of the scattering centers, $\varepsilon$ is the additive noise matrix with the same size as $\mathbf{S}$, and $\mathbf{F}$ is a $\bar{N} \times M$ partial Fourier matrix given by

$$
\mathbf{F}=\left[\begin{array}{llll}
1 & 1 & \cdots & 1 \\
1 & \omega & \cdots & \omega^{(N-1)} \\
\vdots & \vdots & \ddots & \vdots \\
1 & \omega^{(N-1)} & \cdots & 1
\end{array}\right]_{N \times N} \text { and } \omega=\exp \left(-j \frac{2 \pi}{\bar{N}}\right)
$$

Notably, matrix $\mathbf{F}$ can be regarded as an over-completed dictionary, which bridges the recorded data and the SR image. It should be emphasized that the above signal model is based on two significant assumptions.

1) Complete motion compensation. In (2), only the additive noise is taken into account while other model errors such as range shift and residual phase error are assumed removed. There are many precise motion compensation algorithms [35-41] applicable making this assumption rigid.

2) Stationarity assumption. The signal amplitude and Doppler frequency are assumed to be invariant. In this paper, we focus on the SR imaging with short CPI, during which the signal can be regarded as stationary and involves no time variance of both the reflectivity and Doppler for a scatterer.

B. Bayesian SR (BSR) imaging via independent and identical (IID) statistics assumption

Generally, the components of $\varepsilon$ are approximated as a zero-mean complex Gaussian noise, namely, its imaginary and real parts (denoted by $\boldsymbol{\varepsilon}_{r}$ and $\boldsymbol{\varepsilon}_{i}$ ) are subject to independent zero-mean Gaussian distribution. The complexvalued normal probability density function (PDF) of $\varepsilon$ with unknown variance $\sigma^{2}$ is given by

$$
p\left(\boldsymbol{\varepsilon} \mid \sigma^{2}\right)=\left(\pi \sigma^{2}\right)^{-N \cdot M} \exp \left(-\frac{1}{\sigma^{2}}\|\boldsymbol{\varepsilon}\|_{F}^{2}\right)
$$

where $\|\cdot\|_{F}^{2}$ denotes the square of the Frobenius norm of a matrix (the $\|\mathbf{M}\|_{F}^{2}$. Notation for a matrix $\mathbf{M}=\left[b_{n m}\right]_{N \times M}$ denotes $\left.\|\mathbf{M}\|_{F}^{2}=\sum_{n, m}\left|b_{n m}\right|^{2}\right)$. Therefore, we have the Gaussian likelihood function of the observation, which is

$$
p\left(\mathbf{S} \mid \mathbf{A}, \sigma^{2}\right)=\left(\pi \sigma^{2}\right)^{-N \cdot M} \exp \left(-\frac{1}{\sigma^{2}}\|\mathbf{S}-\mathbf{F A}\|_{F}^{2}\right)
$$

ISAR imagery demonstrates the spatial distribution of the target scattered field in the RD plane. Dominant scattering centers usually occupy only a fraction of the whole RD bins even though they contribute a majority of the energy, while signals from weak scattering centers contribute little to image formation. This sparse characteristic of ISAR images can be exploited to achieve SR. In this section, we develop a SR approach by combining Bayesian statistics and 
compressive sensing theory. According to sparse Bayesian learning (SBL) [42] and BCS [33], the sparsity can be formulized by placing a sparseness-promoting prior on $\mathbf{A}$. In general, a Laplace probability distribution can be used to enforce the sparsity prior on the recovered objective signal when the signal is real valued [42, 43]; however, it is not straightforward to extend the Laplace distribution to the complex-valued model. In the following content, an extension of the two-layer SBL model [42] is presented and a Laplace distribution for complex signal is developed to encourage sparse representation of $\mathbf{A}$.

Let $a_{n m}=r_{n m}+j \cdot i_{n m}$, where $r_{n m}$ and $i_{n m}$ denote the real and imagery part of $a_{n m}$, respectively. Then the PDF of $a_{n m}$ can be expressed by the joint PDF of $r_{n m}$ and $i_{n m}$. Following the two-layer SBL probabilistic framework [42], we assume both the real and imaginary parts independently follow the same zero-mean Gaussian distribution with variance $\beta$. Therefore, the PDF of $a_{n m}$ has a complex Gaussian form as

$$
\begin{aligned}
p\left(a_{n m} \mid \beta\right) & =p\left(r_{n m}, i_{n m} \mid \beta\right) \\
& =\frac{1}{\pi \beta} \exp \left(-\frac{r_{n m}^{2}+i_{n m}^{2}}{\beta}\right)
\end{aligned}
$$

In the two-layer hierarchical prior model of SBL, a hyperprior PDF for $\beta$ is introduced. The gamma distribution is usually used to induce the sparsity properties of the reconstruction [42], that is

$$
p(\beta \mid a, b)=\mathbf{G a m m a}(\beta \mid a, b)=\frac{b^{a}}{\boldsymbol{\Gamma}(a)} \beta^{a-1} \exp (-b \cdot \beta)
$$

where $\operatorname{Gamma}(\cdot \mid a, b)$ denotes a gamma PDF with shape parameter $a$ and rate parameter $b$. A detailed analysis on parameter selection for this PDF can be found in [44]. Integrating out $\beta$, we have

$$
\begin{gathered}
p\left(a_{n m} \mid a, b\right)=\int_{0}^{\infty} p\left(a_{n m} \mid \beta\right) p\left(\delta_{2} \mid \gamma_{2}\right) d \beta \\
=\int_{0}^{\infty} \frac{1}{\pi \beta} \exp \left(-\frac{r_{n m}^{2}+i_{n m}^{2}}{\beta}\right) \cdot \frac{b^{a}}{\Gamma(a)} \beta^{a-1} \exp (-b \cdot \beta) d \beta
\end{gathered}
$$

Following the two-layer SBL probabilistic framework [33, 42, 43], we set $a=\frac{3}{2}$

$$
\begin{aligned}
p\left(a_{n m} \mid \frac{3}{2}, b\right) & =\int_{0}^{\infty} \frac{1}{\pi \beta} \exp \left(-\frac{r_{n m}^{2}+i_{n m}^{2}}{2 \beta}\right) \cdot \frac{b^{\frac{3}{2}}}{\Gamma\left(\frac{3}{2}\right)} \beta^{\frac{1}{2}} \exp (-b \cdot \beta) d \beta \\
& =\frac{b^{\frac{3}{2}}}{\pi \cdot \Gamma\left(\frac{3}{2}\right)} \int_{0}^{\infty} \beta^{-\frac{1}{2}} \exp \left(-\frac{r_{n m}^{2}+i_{n m}^{2}}{2 \beta}-b \cdot \beta\right) \cdot d \beta \\
& =\frac{b^{\frac{3}{2}}}{\pi \cdot \Gamma\left(\frac{3}{2}\right)} \int_{0}^{\infty} \exp \left(-\frac{r_{n m}^{2}+i_{n m}^{2}}{2 \beta}-b \cdot \beta\right) d \beta^{\frac{1}{2}}
\end{aligned}
$$

By using the relation $\int_{0}^{\infty} \exp \left[-\frac{1}{2}\left(h^{2} u^{2}+k^{2} u^{-2}\right)\right] d u$ $=\sqrt{\frac{\pi}{2 h^{2}}} \exp (-|h k|)$ [45], the PDF of $a_{n m}$ is developed as a function of $b$, which is given by

$$
p\left(a_{n m} \mid b\right)=\frac{b}{\pi} \exp \left(-\sqrt{2 b}\left|a_{n m}\right|\right)
$$

Setting $\gamma=\sqrt{2 b}$, (9) becomes

$$
p\left(a_{n m} \mid \gamma\right)=\frac{\gamma^{2}}{2 \pi} \exp \left(-\gamma\left|a_{n m}\right|\right)
$$

Supposing the image pixels are subject to the IID PDF in (10), we have the likelihood function of $A$ as

$$
\begin{aligned}
p(\mathbf{A} \mid \gamma) & =\prod_{n=0, m=0}^{\bar{N}-1, M-1}\left(\frac{\gamma^{2}}{2 \pi}\right) \exp \left(-\gamma\left\|a_{n m}\right\|_{1}\right) \\
& =\left(\frac{\gamma^{2}}{2 \pi}\right)^{\bar{N} \cdot M} \cdot \exp \left(-\gamma\|\mathbf{A}\|_{1}\right)
\end{aligned}
$$

where $\|\mathbf{A}\|_{1}=\sum_{n=1}^{\bar{N}} \sum_{m=1}^{M}\left\|a_{n m}\right\|_{1}$. Then, reconstructing SR image is shifted into a classical problem of estimating A from noisy observation $\mathbf{S}$, which has the MAP estimator

$$
\hat{\mathbf{A}}(\mathbf{S})=\arg \max _{\mathbf{A} \in \mathbf{C}_{\bar{N} \times M}}[p(\mathbf{A} \mid \mathbf{S})]
$$

By Bayes rule, we get

$$
\hat{\mathbf{A}}(\mathbf{S})=\arg \max _{\mathbf{A} \in \mathbf{C}_{\bar{N} \times M}}\left[p\left(\mathbf{S} \mid \mathbf{A}, \sigma^{2}\right) \cdot p(\mathbf{A} \mid \gamma)\right]
$$

Which is equivalent to

$$
\hat{\mathbf{A}}(\mathbf{S})=\arg \max _{\mathbf{A} \in \mathbf{C}_{\bar{N} \times M}}\left\{\log \left[p\left(\mathbf{S} \mid \mathbf{A}, \sigma^{2}\right)\right]+\log [p(\mathbf{A} \mid \gamma)]\right\}
$$

Substituting (4) and (11) into (14), the MAP estimator becomes

$$
\begin{gathered}
\hat{\mathbf{A}}(\mathbf{S})=\arg \max _{\mathbf{A} \in \mathbf{C}_{\tilde{N} \times N}}\left\{-\frac{1}{\sigma^{2}}\|\mathbf{S}-\mathbf{F A}\|_{F}^{2}-\gamma\|\mathbf{A}\|_{1}\right\} \\
=\arg \min _{\mathbf{A} \in \mathbf{C}_{\tilde{N} \times N}}\left\{\|\mathbf{S}-\mathbf{F A}\|_{F}^{2}+\mu\|\mathbf{A}\|_{1}\right\}
\end{gathered}
$$

where $\mu=\sigma^{2} \gamma$ is defined as the sparsity coefficient which is directly related to the unknown statistics of noise and the target signal. One can note that the optimization composes two different terms: the $l_{2}$-norm preserves the data fidelity of the solution and the $l_{1}$-norm imposes most elements of $\mathbf{A}$ to be small with a few large ones in accordance with its spatial sparsity. The complex-valued Laplace distribution model in (11) can be also applied in other complex-valued imaging problems, such as terahertz imaging [46]. Clearly, MAP estimator of a SR 
image corresponds to a $l 1$-regularized optimization which is often referred to as the basis pursuit de-noising (BPDN) problem [47]. It is also widely accepted that the problem in (15) is also equivalent to the $l_{1}$-constrained optimization in CS [48]; however, BSR imaging has evident differences with them. Compared to the pointenhanced algorithm [26], the $l_{1}$-norm weighting coefficient in (15) is explicitly associated with the noise and target statistics in a Bayesian sense. Furthermore, different from the CS-based SR imaging in [29], BSR is closely connected to convex quadratic programming [49] making it very efficient and accurate.

\section{Improved Bayesian SR (IBSR) imaging based on nonidentical statistics assumption}

In the BSR optimization (15), all the components of A are treated equally as they are assumed to follow the same Laplace distribution. In reality, the scattered field of a distributed target usually has the spatial-assembling feature corresponding to the phenomenon that most significant scattering centers are concentrated around a small region in the RD image. Neighboring pixels tend to be large or small simultaneously, characterizing themselves with a specific spatial organization. In this sense, placing the identical Laplace distribution on all components of the image would ignore both the spatial configuration and the energy-assembling feature of the target scattering filed. Nevertheless, it is of great potential to substantially improve the performance of BSR imaging by leveraging statistics models that better suit the target image. In order to respect the local spatial organization and discriminate different elements of the image statistically, we introduce nonidentical Laplace distributions for each component of the ISAR image. Following the definition in (10), for the specific component $a_{n m}=\mathbf{A}(n, m)$, the Laplace density function is rewritten as

$$
p\left(a_{n m} \mid \gamma_{n m}\right)=\left(\frac{\gamma_{n m}^{2}}{2 \pi}\right) \cdot \exp \left(-\gamma_{n m}\left|a_{n m}\right|\right)
$$

Supposing the image pixels are independent, we have PDF of the SR image as

$$
\begin{aligned}
p\left(\mathbf{A} \mid \gamma_{00}, \gamma_{01} \cdots, \gamma_{(\bar{N}-1)(M-1)}\right) & =\prod_{m=0}^{M-1} \prod_{n=0}^{\bar{N}-1}\left(\frac{\gamma_{n m}^{2}}{2 \pi}\right) \exp \left(-\gamma_{n m}\left|a_{n m}\right|\right) \\
& =\prod_{m=0}^{M-1}\left[\prod_{n=0}^{\bar{N}-1}\left(\frac{\gamma_{n m}^{2}}{2 \pi}\right)\right] \exp \left(-\left\|\mathbf{W}_{m} \mathbf{a}_{m}\right\|_{1}\right)
\end{aligned}
$$

where $\mathbf{a}_{m}=\mathbf{A}(:, m)$ corresponds to the $m$ th column of the RD image and $\mathbf{W}_{m}=\operatorname{diag}\left[\begin{array}{llll}\gamma_{0 m} & \gamma_{1 m} & \cdots & \gamma_{(\bar{N}-1) m}\end{array}\right]_{\bar{N} \times \bar{N}}$ is the diagonal matrix corresponding to the Laplace distributions. Introducing nonidentical scale parameters in (20) provides immediate benefits. We want the probabilistic model to favor certain configurations for the magnitudes and locations of the significant scattering centers that promote the performance of the BSR imaging. For this purpose, a long tailed distribution with small scale parameter for each prominent scattering center imposes high probability of a large-valued pixel, and large scale parameters for other signal components lead to small value prediction. The MAP estimator is then given by

$$
\hat{\mathbf{A}}(\mathbf{S})=\arg \max _{\mathbf{A} \in \mathbf{C}_{\bar{N} \times M}}\left[p\left(\mathbf{S} \mid \mathbf{A}, \sigma^{2}\right) \cdot p\left(\mathbf{A} \mid \gamma_{00}, \gamma_{01} \cdots, \gamma_{(\bar{N}-1)(M-1)}\right)\right]
$$

Which is also equivalent to

$$
\begin{aligned}
\hat{\mathbf{A}}(\mathbf{S}) & =\arg \max _{\mathbf{A} \in \mathbf{C}_{\bar{N} \times M}}\left\{\log \left[p\left(\mathbf{S} \mid \mathbf{A}, \sigma^{2}\right)\right]\right. \\
& \left.+\log \left[p\left(\mathbf{A} \mid \gamma_{00}, \gamma_{01} \cdots, \gamma_{(\bar{N}-1)(M-1)}\right)\right]\right\}
\end{aligned}
$$

Substituting Eqs. (4) and (17) into (19), the MAP estimator becomes

$$
\begin{gathered}
\hat{\mathbf{A}}(\mathbf{S})=\arg \max _{\mathbf{A} \in \mathbf{C}_{\tilde{N} \times M}}\left\{-\frac{1}{\sigma^{2}}\|\mathbf{S}-\mathbf{F A}\|_{F}^{2}-\sum_{m=0}^{M-1}\left\|\mathbf{W}_{m} \mathbf{a}_{m}\right\|_{1}\right\} \\
=\arg \min _{\mathbf{A} \in \mathbf{C}_{\bar{N} \times M}}\left\{\sum_{m=0}^{M-1}\left(\left\|\mathbf{s}_{m}-\mathbf{F} \mathbf{a}_{m}\right\|_{F}^{2}+\sigma^{2}\left\|\mathbf{W}_{m} \mathbf{a}_{m}\right\|_{1}\right)\right\}
\end{gathered}
$$

where $\mathbf{s}_{m}=\mathbf{S}(:, m)$ denotes the $m$ th column of $\mathbf{S}$ corresponding to $\mathbf{a}_{m}$. In contrast to BSR in (15), the image is modeled by the nonidentical Laplace distribution in (20). We designate it improved BSR (IBSR). IBSR imaging can discriminate pixels containing the strong scattering centers from the weak ones, which emphasizes the spatial configuration and strong components in image formation. To this end, each element of the image is treated individually to favor the recovery of the structural organization of the target scattered field. To encourage the probability of scattering center reconstruction, and enforce the noise be near zero, small scale parameters are preferable for scattering centers, while large scale parameters for noise components. Furthermore, similar to the optimization in weighted compressive sensing [50], the weights in the $l_{1}$-norm penalty term in (20) will influence the contributions of different components to the penalty function improving reconstruction accuracy and efficiency. Therefore, if proper scale parameters are utilized in (20), different distributions of signal and noise can be obtained, leading to more precise signal recovery and more effective noise suppression. 


\section{Statistics estimation and imaging solver}

\section{A. Statistics estimation and imaging procedure}

It is widely accepted that in the Bayesian optimization in (15), if $\mu$ is set too large, weak scatterers together with noise will be rejected in the reconstruction of image leaving only the dominant scattering centers. If $\mu$ is too small, then a large amount of noise may be tolerated in the image. Therefore, for successful SR imaging via the MAP estimator in (15), we must choose $\sigma^{2}$ and $\gamma$ optimally. In ISAR imaging, strong scattering centers of the target are usually clustered densely in only a small fraction of the whole RD plane. Therefore, some pure noise pixels are evidently distinguishable from the target support in some range bins, with which the noise variance can be estimated straightforwardly. For the other factor $\gamma$, supplementary approaches can be employed. As is known, conventional SR methods are usually precise and robust enough to extrapolate the short aperture to a limited length, such as twice the original aperture length. In real applications, we find that in the BSR optimization, using $\gamma$ estimated from the SR image generated by Burg's BWE performs well when its SR factor is small. This motivates us to combine BWE techniques as an auxiliary tool to provide a coarse SR image to determine the statistical parameters for constructing the Bayesian SR images. In this section, we design a scheme for parameter estimation by combining the BWE and CFAR detection techniques together with a stage-by-stage procedure to achieve a desired SR robustly. The main characteristic of this process is extrapolation of aperture of the signal by a factor of two and updating the estimate of the Laplace scale parameter each stage. In this procedure, the scale parameter estimation depends on the SR image with double BWE from last stage, yielding better BSR and IBSR images. Therefore, the resolution is enhanced step by step until a desired SR is achieved. The procedure runs in the following three steps:

1) We apply Fourier transform (FT) to the rangecompressed data to generate the coarse RD image and estimate the noise variance by using the pure noise samples. Those noise samples are also used to develop a CFAR threshold to remove noise in the background of the coarse image. It should be emphasized that the noise within the target region cannot be removed; however, due to the high SNR gain from two-dimensional coherent integration, this noise does not affect much. The de-noised image is then transformed back into the time domain by an inverse Fourier transform (IFT). This is followed by Burg's BWE to extrapolate the aperture twice, and then the scale parameter is estimated from the BWE image based on the ML rule. This step functions as the initialization.

2) The Bayesian optimization for an image with a SR factor of two is developed with the statistics from the last step and then solved by a Quasi-Newton solver (this solver will be introduced in the following section). The reconstructed SR image is transformed back into the time domain by IFT, after which, Burg's BWE with double extrapolation is employed to obtain an image with SR at a factor of four and to estimate the scale parameter for the next stage.

3) We repeat the second step until a desired SR is reached.

Step 1 plays a significant role in determining the noise variance and coarse target support in the RD plane. With suitable motion compensation, a RD image of size $N \times M$ is obtained by a FT in the azimuth direction. The statistical parameters can be estimated by using the RD image. Since motion compensation usually removes all translational motion, the target is centralized in the RD plane, and thus, the pixels corresponding to high Doppler frequency contain noise only. In practice, we usually use the range bins excluding the target support as the pure noise samples. These pure noise samples can be used to form the ML estimate of $\sigma^{2}$. Suppose that we choose $N_{\varepsilon}$ pure noise samples and place them in a vector with real and imaginary parts given by $\left[\varepsilon_{r}(n)\right]_{N_{\varepsilon} \times 1}$ and $\left[\varepsilon_{i}(n)\right]_{N_{\varepsilon} \times 1}$, respectively, the ML estimate $\hat{\sigma}^{2}$ of $\sigma^{2}$ is given by

$$
\hat{\sigma}^{2}=\frac{1}{N_{\varepsilon}}\left[\sum_{n=0}^{N_{\varepsilon}} \varepsilon_{r}^{2}(n)+\sum_{n=0}^{N_{\varepsilon}} \varepsilon_{i}^{2}(n)\right]
$$

This estimate is usually accurate due to the abundance of noise samples. The CFAR detector for strong scattering centers in the $\mathrm{RD}$ image can be developed subsequently. The RD image has high SNR gain from the two-dimension coherent integration, and so strong scattering centers are discernible from noise in amplitude. In general, discriminating strong scatterers from noise in the RD domain corresponds to distributed target detection under homogeneous Gaussian noise. Noting $\hat{\sigma}^{2}$ also corresponds to the power of noise, a CFAR threshold is developed as [51].

$$
T=\sqrt{\hat{\sigma}^{2} \cdot \lambda}
$$

where $\lambda$ is a scale coefficient used to achieve a desired constant false alarm probability. For an extensive mathematical study of the CFAR detector, see [34] and [51]. To calculate $\lambda$, the software provided by Glen Davidson [52] and Matlab function npwgnthresh.m can be used directly. Pixels with amplitude larger than the CFAR 
threshold are determined as target components, while those below the threshold are classified as noise and set to zeros to achieve the de-noised image. Only the noise components in the background region are detected and removed, while noise mixed with the target region remains. However, due to the high SNR gain from the double coherent integration, the remaining noise has little affect. The de-noised image is then transformed back into the time domain where Burg's BWE is used to extrapolate the data doubly. Therefore, a SR image can be achieved by a FT after Burg's BWE processing. For clarity, we define $\overline{\mathbf{A}}_{1}=\left[\overline{\mathrm{A}}_{1}(n, m)\right]_{N_{1} \times M}$ as the BWE image with size $N_{1} \times M\left(N_{1}=2 N\right)$. Then, the parameter scale $\gamma_{1}$ of the image with SR at a factor of two is ready to be estimated by the ML rule. The ML estimator of $\gamma_{1}$ is formed by finding $\gamma_{1}$ that maximizes the log-likelihood function

$$
\log \left[\boldsymbol{P}\left(\overline{\mathbf{A}}_{1} \mid \gamma_{1}\right)\right]=\log \left[\left(\frac{\gamma_{1}^{2}}{2 \pi}\right)^{N_{1} \cdot M} \cdot \exp \left(-\gamma_{1}\left\|\overline{\mathbf{A}}_{1}\right\|_{1}\right)\right]
$$

Differentiating with respect to $\gamma_{1}$ yields

$$
\frac{\partial \log \left[\boldsymbol{P}\left(\overline{\mathbf{A}}_{1} \mid \gamma_{1}\right)\right]}{\partial \gamma_{1}}=\frac{2 N_{1} \cdot M}{\gamma_{1}}-\sum_{n=0}^{N_{1}-1} \sum_{m=0}^{M-1}\left|\overline{\mathbf{A}}_{1}(n, m)\right|
$$

Setting (24) equal to zero so that $\gamma_{1}$ is a critical point leads to the ML estimator

$$
\hat{\gamma}_{1}=\frac{2 N_{1} \cdot M}{\sum_{n=0}^{N_{1}-1} \sum_{m=0}^{M-1}\left|\overline{\mathrm{A}}_{1}(n, m)\right|}
$$

This shows that the ML estimator of $\gamma_{1}$ is the reciprocal of the amplitude mean of $\overline{\mathbf{A}}_{1}$. Without loss of generality, in the initialized step, all variables are indicated by the subscript $g=1$, and the initialization step is embedded in the first stage of the whole procedure.

Steps 2 and 3 implement the BSR imaging in an iterative manner. By using $\hat{\sigma}^{2}$ and $\hat{\gamma}_{1}$, the optimization for a SR image with double aperture extrapolation which corresponds to the first stage of SR imaging can be developed. For clarity, we use subscript $g$ as the stage number, and in the first stage, we have $g=1$. BSR optimization in the gth stage is given by

$$
\hat{\mathbf{A}}_{g}=\arg \min _{\mathbf{A} \in \mathbf{C}_{\bar{N}_{g} \times M}}\left\{\left\|\mathbf{S}-\mathbf{F}_{g} \mathbf{A}_{g}\right\|_{F}^{2}+\hat{\sigma}^{2} \hat{\gamma}_{g}\left\|\mathbf{A}_{g}\right\|_{1}\right\}
$$

where $\mathbf{A}_{g}$ denotes the $\bar{N}_{g} \times M\left(\bar{N}_{g}=2^{g} N\right)$ SR image, and the $N \times \bar{N}_{g} \mathbf{F}_{g}$ can be calculated by using Eq. (3). Similarly, Burg's BWE is applied to $\hat{\mathbf{A}}_{g}$ in order to estimate the Laplace scale parameter for the next stage. We transform $\hat{\mathbf{A}}_{g}$ back into the azimuth time domain and use Burg's BWE to generate the $\bar{N}_{g+1}$ $\times M$ BWE image $\overline{\mathbf{A}}_{g+1}=\left[\overline{\mathrm{A}}_{g+1}(n, m)\right]_{N_{1} \times M}$ which is then used in the ML estimation of the next Laplace scale parameter $\hat{\gamma}_{g+1}=\frac{2 N_{g+1} \cdot M}{\sum_{n=0}^{N_{g+1^{-1}}} \sum_{m=0}^{M-1}\left|\overline{\mathrm{A}}_{g+1}(n, m)\right|}$. The BSR optimization for the next stage can be performed using $\hat{\gamma}_{g+1}, \hat{\sigma}^{2}$, and $\mathbf{F}_{g+1}$. We increment $g$ and repeat this processing until a desired SR is obtained. For example, if we want to achieve a SR factor of eight, we need three stages for BSR imaging together with the initialization step, and in each stage, we need to perform the Burg's BWE and BSR imaging once. For a clear description of the procedure, we list the corresponding variables in Table 1 and give a detailed flowchart of the BSR with G stages in Fig. 1.

It should be noted that the Burg's BWE plays an essential role in the estimation of the Laplace scale parameter. Implemented in a recursive way, Burg's BWE is stable in aperture extrapolation. In real data processing, the order of the linear prediction model in the BWE is typically set high, such as one third of the data length, to ensure estimation precision of the largest coefficients without bringing spurious scattering centers [10]. Additionally, in the Burg's BWE, the retention of primary data and phase coherency of the extrapolated signal are meaningful for the precise estimation of the scale parameter. However, in [53], detailed theoretic analysis reveals that Burg's BWE has limited SR ability at a factor of 2.6 for two identical point-scatterers, and beyond that, the negative effect becomes evident, which is the main reason why the extrapolation factor of two in each stage is set.

Similarly, IBSR imaging with the optimization in (20) can be also performed by using the parameter estimation and stage-by-stage procedure presented above. Unlike BSR imaging, IBSR utilizes the nonidentical Laplace distribution, and the estimation of the scale parameters should be reconsidered. As mentioned before, the ISAR image represents the local spatial structure and energyassembling characteristic of the target scattering field, which means that neighboring components tend to be large or small simultaneously. Therefore, we estimate the scale parameter corresponding to a certain component by

Table 1 Variable description in the stage-by-stage procedure

\begin{tabular}{ll}
\hline$g$ & Subscript of the stage number \\
$\bar{N}_{g}=2^{g} \cdot N$ & Azimuth dimension of SR image in the gth stage \\
$\mathbf{A}_{g}$ & SR image $\left(\bar{N}_{g} \times M\right)$ \\
$\hat{\mathbf{A}}_{g}$ & Estimation of $\mathbf{A}_{g}\left(\bar{N}_{g} \times M\right)$ \\
$\overline{\mathbf{A}}_{g}$ & BWE SR image $\left(\bar{N}_{g} \times M\right)$ in the gth stage \\
$\mathbf{F}_{g}$ & Partial Fourier matrix $\left(\bar{N}_{g} \times N\right)$ \\
\hline
\end{tabular}




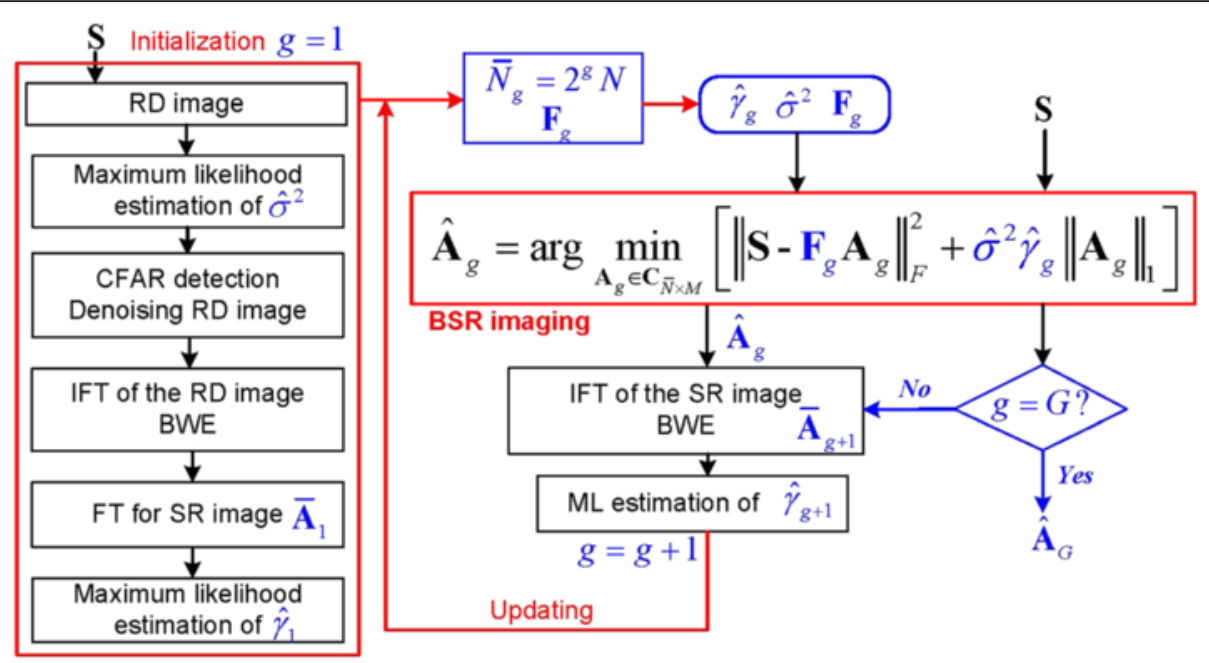

Fig. 1 Flowchart of BSR imaging

using its neighbors, such as the $5 \times 5$ neighborhood surrounding it. It should be noted that in reality, the number of the neighboring components should be selected optimally since if we use only a few neighboring pixels, severe deviation would be introduced in the result, while if we use a large number of neighbors, they would tend to not follow similar distributions leading to poor IBSR performance as well. A useful scheme is to use only those with similar magnitudes to the central pixel in a small neighboring window 11 (such as $3 \times 3$ ) in the first stages, and in the last stages select those with similar magnitudes in a large window (such as $5 \times 5$ ) to estimate the Laplace scale for the central pixel. The scale parameter is estimated as the reciprocal of the mean of these pixel values similar to the ML estimation in (25). With the help of CFAR detection and Burg's BWE, the resolution is enhanced stage-by-stage until it reaches the predetermined factor. Similarly, for the gth stage, we have the IBSR optimization as

$$
\hat{\mathbf{A}}_{g}=\arg \min _{\mathbf{A} \in \mathbf{C}_{N_{g} \times M}}\left\{\sum_{m=0}^{M-1}\left(\left\|\mathbf{s}_{m}-\mathbf{F}_{g} \mathbf{a}_{m(g)}\right\|_{F}^{2}+\hat{\sigma}^{2}\left\|\mathbf{W}_{m(g)} \mathbf{a}_{m(g)}\right\|_{1}\right)\right\}
$$

For clarity, a flowchart for IBSR imaging with G stages is provided in Fig. 2.

\section{B. An effective solver to the IBSR optimization}

As mentioned in the above section, many algorithms and software [54-57] are ready to be applied in order to solve the optimizations of (15) and (20). Herein, we apply a Quasi-Newton solver to them. This takes advantage of the fast Fourier transform




(FFT), promising high efficiency and the possibility of real-time implementation of BSR and IBSR imaging. In the following content, we only deduce the solver to the optimization (20), but it can be extended to (15) straightforwardly. Noting that the optimizations in (15) and (20) are non-differentiable, we need to modify them to be consistent with the gradient-based optimization algorithms. In order to overcome the non-differentiability of the $l_{1}$-norm around the origin in (20), a useful approximation $[27,58]$ is employed by

$$
\left\|a_{n m}\right\|_{1} \approx\left(\left|a_{n m}\right|^{2}+\tau\right)^{1 / 2}
$$

where $|\cdot|$ stands for the modulus operator, and $\tau$ is a small nonnegative parameter. To ensure the approximation as rigid as possible, $\tau$ should be set small. The IBSR optimization in (18) can be reformatted as

$$
\begin{aligned}
\hat{\mathbf{A}}(\mathbf{S})= & \arg \min _{\mathbf{A} \in \mathbf{C}_{\bar{N} \times M}}\left\{\sum _ { m = 0 } ^ { M - 1 } \left(\left\|\mathbf{s}_{m}-\mathbf{F} \mathbf{a}_{m}\right\|_{F}^{2}\right.\right. \\
& \left.\left.+\sigma^{2} \sum_{n=0}^{\bar{N}-1} \gamma_{n m} \cdot\left(\left|a_{n m}\right|^{2}+\tau\right)^{1 / 2}\right)\right\}
\end{aligned}
$$

The approximation in (28) keeps the optimizations in (20) and (29) consistent, and the solution to (29) tends to that of the optimization (20) as $\tau$ approaches to zero. Therefore, a precise solution to optimization (20) can be achieved by solving (29) if and only if a very small $\tau$ is used in (28). In the following experiments, $\tau=10^{-6}$. The selection of $\tau$ is still an open problem, and it is empirically small to get rid of large approximation errors. Due to the independence between range cells, solving the two-dimensional optimization (29) is equivalent to solving the onedimension optimization.

$$
\hat{\mathbf{a}}_{m}(\mathbf{S})=\arg \min _{\mathbf{a}_{m} \in \mathbf{C}_{\bar{N} \times 1}}\left\|\mathbf{s}_{m}-\mathbf{F} \mathbf{a}_{m}\right\|_{F}^{2}+\sigma^{2} \sum_{n=0}^{\bar{N}-1} \gamma_{n m} \cdot\left(\left|a_{n m}\right|^{2}+\tau\right)^{1 / 2}
$$

for each range cell separately. That is, in order to reconstruct the $m$ th column of A, we must solve (30).

The conjugate gradient of $f\left(\mathbf{a}_{m}\right)=\left\|\mathbf{s}_{m}-\mathbf{F} \mathbf{a}_{m}\right\|^{2}+2 \sigma^{2}$

$\sum_{n=0}^{\bar{N}-1} \mathbf{W}_{m}(n, n) \cdot\left(\left|a_{n m}\right|^{2}+\tau\right)^{1 / 2}$ with respect to $\mathbf{a}_{m}$ is given by

$$
\begin{aligned}
\nabla_{\mathbf{a}_{m}} f\left(\mathbf{a}_{m}\right)= & 2 \mathbf{F}^{H} \mathbf{F} \mathbf{a}_{m}+\sigma^{2} \cdot \mathbf{U}\left(\mathbf{a}_{m}\right) \cdot \mathbf{W}_{m} \mathbf{a}_{m}-\mathbf{F}^{H} \mathbf{s}_{m} \\
= & \mathbf{H}\left(\mathbf{a}_{m}\right) \cdot \mathbf{a}_{m}-\mathbf{F}^{H} \mathbf{s}_{m}
\end{aligned}
$$

where the Hessian matrix $\mathbf{H}\left(\mathbf{a}_{m}\right)$ is approximately given by

$$
\mathbf{H}\left(\mathbf{a}_{m}\right) \approx 2 \mathbf{F}^{H} \mathbf{F}+\sigma^{2} \cdot \mathbf{U}\left(\mathbf{a}_{m}\right) \cdot \mathbf{W}_{m}
$$

and

$$
\begin{aligned}
& \mathbf{U}\left(\mathbf{a}_{m}\right)=\operatorname{diag}\left[1 /\left(\left|a_{0 m}\right|^{2}+\tau\right)^{1 / 2}, 1 /\left(\left|a_{1 m}\right|^{2}+\tau\right)^{1 / 2}, \cdots,\right. \\
& \left.1 /\left(\left|a_{(\bar{N}-1) m}\right|^{2}+\tau\right)^{1 / 2}\right]
\end{aligned}
$$

Because the Hessian approximation relies on the objective $\mathbf{a}_{m}$, an iterative solver to (30) is presented through:

$$
\hat{\mathbf{a}}_{m}^{(h+1)}=\left[\mathbf{H}\left(\hat{\mathbf{a}}_{m}^{(h)}\right)\right]^{-1} \mathbf{F}^{H} \mathbf{s}_{m}
$$

where $\hat{\mathbf{a}}_{m}^{(h)}$ is the estimator of $\mathbf{A}(:, m)$ in the $h$ th iteration. To accelerate the iteration, the conjugate gradient algorithm (CGA) [59] can be applied to avoid the Hessian matrix inversion in (34). A fixed threshold $\delta_{\mathrm{CG}}$ is used for a complete run of the CGA. Without prior information about A, it is initialized as $\hat{\mathbf{A}}_{0}=\mathbf{F}^{H} \mathbf{S}$. We iterate $h$ until $\left|\hat{\mathbf{a}}_{m}^{(h+1)}-\hat{\mathbf{a}}_{m}^{(h)}\right|_{2} /\left|\hat{\mathbf{a}}_{m}^{(h)}\right|_{2} \leq \rho$, where $\rho$ is a small parameter for the predetermined threshold, or $h$ reaches a predetermined maximum iteration number.

In the following computational cost analysis of the Quasi-Newton solver, we keep track of the multiplications. The main computational cost of the solver for IBSR optimization is in using CGA to iterate (34). One may note that the term $\mathbf{F}^{H} \mathbf{F}$ corresponds to the partial Fourier matrix F, allowing us to use a FFT to efficiently calculate $\mathbf{F}^{H} \mathbf{F} \mathbf{a}_{m}$ in (31). We perform an inverse fast Fourier transform (IFFT) to $\mathbf{a}_{m}$, set the components corresponding to the vacant aperture to zero, and then apply a FFT dramatically reducing the computational load. Let the number of the CGA iterations to solve (34) be $N_{\text {CGA }}$. The computational cost of the FFT and IFFT in solving (34) is $2 N_{\mathrm{CGA}} \bar{N} \log _{2} \bar{N}$ flops. If there are $N_{\mathrm{QN}}$ iterations in the Quasi-Newton solver, the total computation cost is approximately $2 N_{\mathrm{QN}} N_{\mathrm{CGA}} \bar{N} \log _{2} \bar{N}$ flops, while the computational load of calculating matrix inversion through the Cholesky factorization [60] is up to $N_{\mathrm{QN}} \bar{N}^{3} / 3$ flops. Since $N_{\mathrm{CGA}}$ is usually on the order of several tens, the efficiency improvement via FFT and CGA is obvious. In practice, we can start with a relaxed CGA tolerance $\delta_{\mathrm{CG}}$ and reduce it as we iterate the 
Quasi-Newton solver. For further improvements, there are optimization algorithms such as the preconditioned conjugate gradient algorithms [60], which can reduce the number of iterations of the standard CGA.

\section{Performance analysis}

\section{A. Simulation for SR analysis}

In this subsection, we analyze the SR power of BSR and IBSR by comparing them with two compressive sensingbased SR imaging approaches. Namely, we compare BSR and IBSR to the CS [29] and improved CS (ICS) [61] imaging methods under different signal-to-noise ratio (SNR) conditions. All of the methods are sparsity-driven. Both the CS and ICS imaging methods are implemented by the cvx software [56], which usually finds the globally optimal solution to the $l_{1}$-norm constraint problem. Meanwhile, the BSR and IBSR optimizations are solved by the QuasiNewton solver by using the CGA and FFT. It should be emphasized that, as pointed out in [32], the needle-like responses of SR methods tell neither the location accuracy nor capability of resolving two close-located scatterers with limited noisy pulses. In general, the performance of all SR approaches degrades in some degree with the increase in noise. This is due to the strong coherence between Fourier basis vectors, amplitude fluctuation of the scatterers, and the increasing presence of false points at adjacent bins that are usually involved. Since it is difficult to find a theory supporting the SR power comparison, we approach the problem experimentally by analyzing the reconstruction of a signal containing two closely spaced scatterers. Taking both the reconstruction accuracy and the presence of false points into account, we use the Mean Square Error (MSE) and a specified probability of detection $P_{D}$ to evaluate the SR power of different methods. The MSE is defined as

$$
\operatorname{MSE}=\frac{(\mathbf{a}-\hat{\mathbf{a}})^{H} \cdot(\mathbf{a}-\hat{\mathbf{a}})}{\bar{N}}
$$

where $\mathbf{a}$ and $\hat{\mathbf{a}}$ is the ideal and the reconstructed signal, respectively, and $\bar{N}$ denotes the length of the reconstruction. We repeat the SR reconstruction 1000 times with different noise levels and use the average MSE as an evaluating metric.

Assuming that there are only two closely located scatterers, probability of detection corresponds to the hypothesis test:

$\mathrm{H}_{0}$ : There is at most one scatterer present in the reconstruction signal;

$\mathrm{H}_{1}$ : There are exactly two scatterers inside the given

Doppler bins of the reconstruction signal.
Note that we take it as a failure when artifacts occur in the reconstruction, which means that the definition of $P_{D}$ is slightly different from that in [32]. In general, the number of scatterers in the reconstructed signal can be converted into the problem of model selection by some information theory rules before the SR reconstruction [32]. In the manner of signal detection, we utilize the CFAR technique by a predefined threshold to determine the number of signal components. The threshold is calculated according to the added noise variance before the SR reconstruction. The false alarm rate is adjusted experientially according to the given noise variance to maintain the threshold value constant at 0.3 , which is selected optimally based on the performance of IBSR. In the following experiments, bins of the reconstructed signal exceeding the threshold are determined to be signal components and the total number of components is outputted for the hypothesis test.

First, we apply the methods to one-dimensional synthetic signals covering the Doppler range -255 to $256 \mathrm{~Hz}$. All signals contain $N=32$ regularly sampled acquisitions with sample interval 1/512 s, providing a Rayleigh resolution of $16 \mathrm{~Hz}$. Each signal contains two scatterers corresponding to two closely located Doppler points whose amplitudes are equally set to unity and have zero phases. We consider four cases of different distances between the two scatterers to analyze the SR power of the methods. In the four cases, the two scatterers are located at Doppler frequencies of $0 / 8 \mathrm{~Hz}, 0$ / $4 \mathrm{~Hz}, 0 / 2 \mathrm{~Hz}$, and $0 / 1 \mathrm{~Hz}$, respectively. To separate the two scatterers in all cases with the given central 32 samples within the dwell time [-31/512, 32/512] (s), we perform the SR methods with SR factors 2, 4, 8, and 16, corresponding to the reconstructed signal lengths 64, 128, 256, and 512, respectively. Complex-valued Gaussian noise is added in to the synthetic signals to generate SNRs from 0 to $20 \mathrm{~dB}$, wherein SNR is defined as the energy ratio of a single point and the added noise.

For comparison, typical results from the sparsitydriven methods with $\mathrm{SNR}=5 \mathrm{~dB}$ are presented in Fig. 3 . The first and second rows correspond to the results from CS- and ICS-based imaging, while the last two rows show the BSR and IBSR images, respectively. From Fig. 3, we find that reconstructing the ideal signal with only 32 noisy samples is achievable by all sparsity-driven approaches. These approaches work well in cases of relatively high SNRs. We can find that under $\mathrm{SNR}=5 \mathrm{~dB}$, the two scatterers are discriminated optimally and the noise is also suppressed effectively in all cases. Nevertheless, as we increase noise, the performance of each approach is expected to degrade producing fluctuating amplitudes and artifacts. In general, the imaging performance of ICS and IBSR degrades more gracefully than that of CS and BSR. To see this, the MSEs of the 


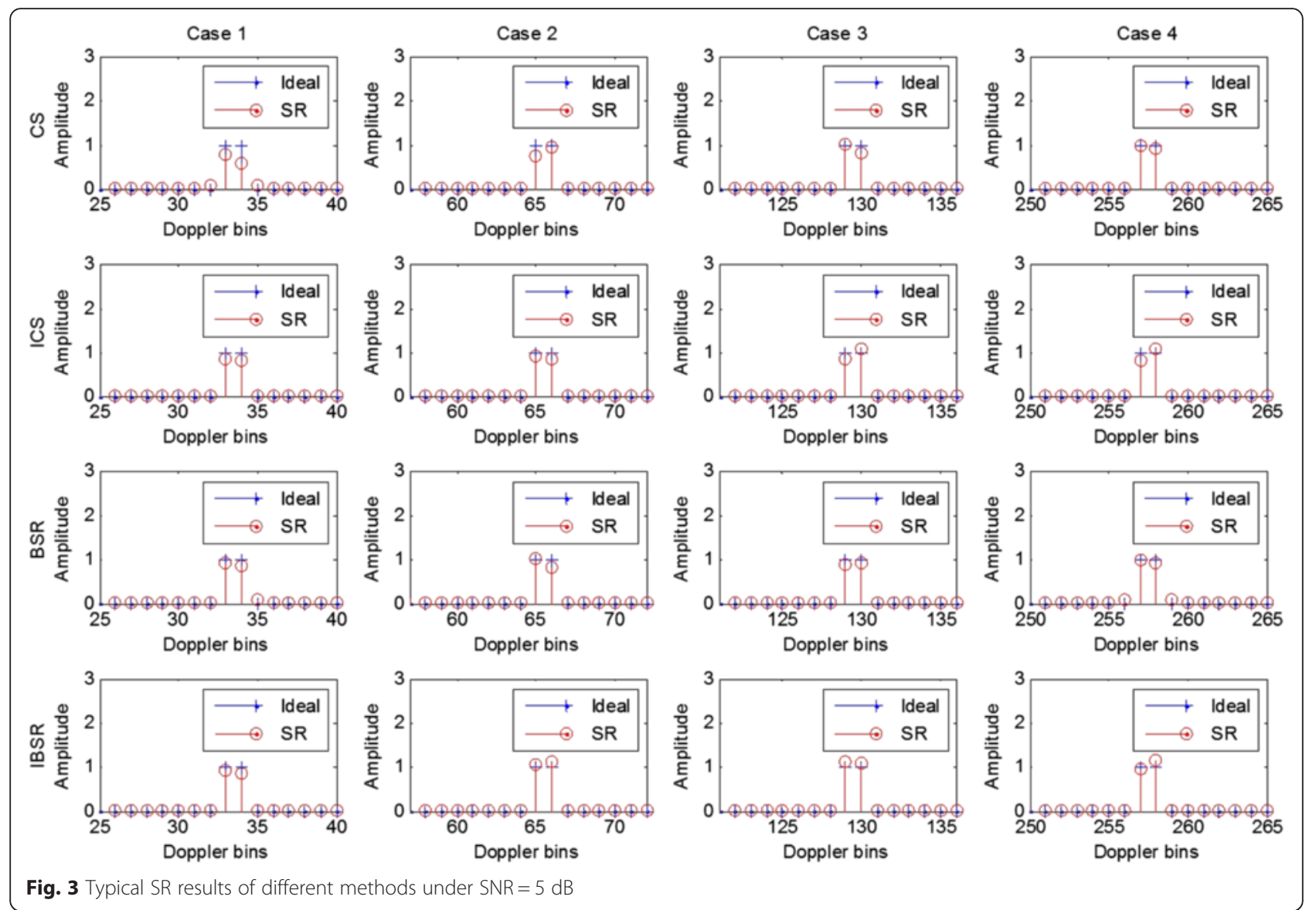

reconstructions are plotted in Fig. 4. From Fig. 4, we note that when the SNR is larger than $10 \mathrm{~dB}$, each method can provide optimal SR reconstruction since they can provide very small MSEs and high probability of detection. Performance degrades with the increase of noise. From Fig. 4, we can note that BSR is comparable to CS-based imaging only in the high SNR situations. This indicates that BSR should be more sensitive to strong noise. In the low SNR cases, ICS and IBSR usually provide better performance than the others, due to the weighting processing in the $l_{1}$-norm optimizations. In particular, IBSR provides the lowest MSE and the highest probability of detection in case 1 and 2 , which demonstrates the improvement achieved by using the nonidentical Laplace probability assumption. However, this performance is not maintained with the increase of SR factor and decrease of SNR. In case 3 and 4, we find that IBSR provides the lowest MSE and the highest probability of detection only in the high SNR situations, with its performance degrading dramatically with the decrease of SNR. Especially in case 3 and 4 when SNR decreases under $5 \mathrm{~dB}$, IBSR is worse than ICS and close to BSR and CS. This is probably due to Laplace scale parameters not being estimated accurately by the proposed iterative procedure due to the strong noise interference and capability limitations of Burg's BWE. It is easy to understand that when MSE increases to an unacceptably high level or the probability of detection decreases under a low threshold, the SR reconstruction can be regarded as a failure, by which one can have a concrete definition of SR. In this sense, ICS and IBSR should be the better options in real applications, and IBSR should be the best from the view of MSE in first and second cases. From the two point-scatterers simulation, both MSE and probability of detection curves provide two perspectives of BSR and IBSR:

1) The performance of BSR can approach cvx-based CS imaging, especially in the cases of relative high resolution. Therefore, although BSR never performs better than CS-based imaging, BSR is preferable in some ideal applications due to its high efficiency.

2) Based on the nonidentical statistical assumption and accommodation of statistical parameters estimation implemented by CFAR and Burg's BWE, IBSR is more robust to noise and limitation of measurements. 
a

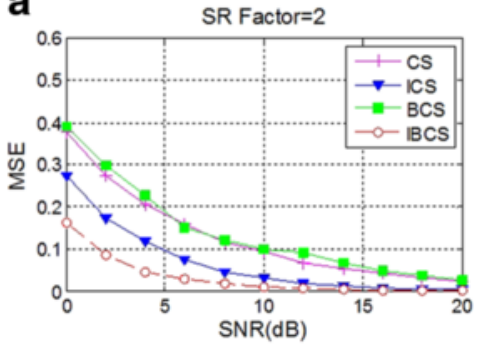

SR Factor $=8$

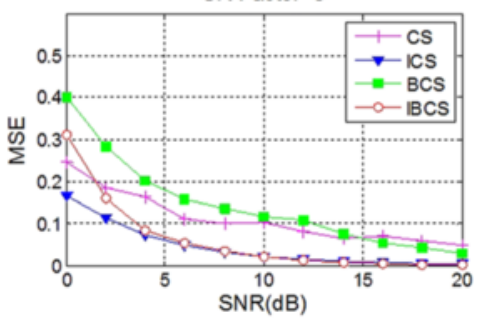

b

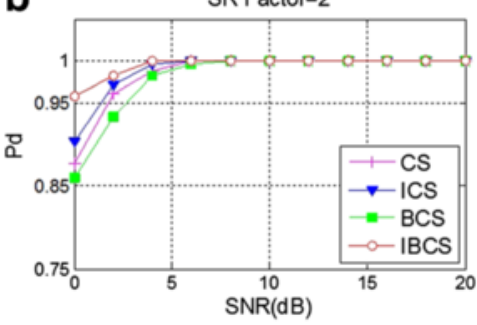

SR Factor $=8$

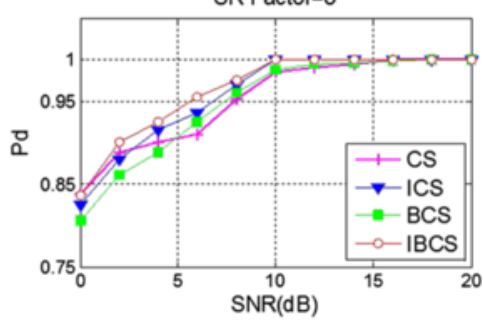

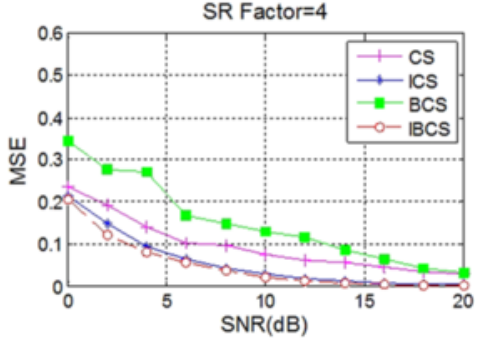

SR Factor $=16$

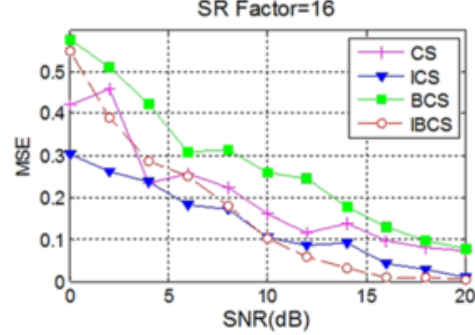

SR Factor $=4$

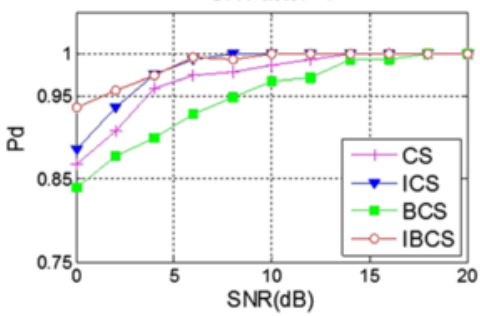

SR Factor $=16$

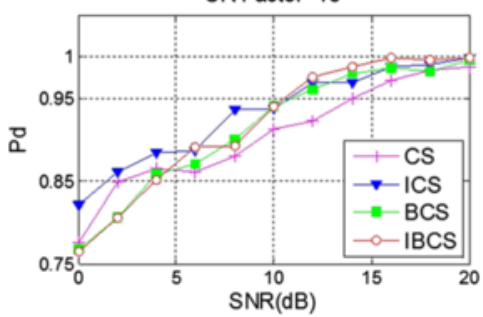

Fig. 4 Comparison of SR performance. a MSEs with different sparsity-driven methods. b $P_{D}$ with different sparsity-driven methods

However, when the SR factor increases up to 8 and 16, performance degrades dramatically. The reason for this phenomenon lies in that, in these cases, Burg's BWE cannot provide accurate estimation of the nonidentical Laplace scale parameters. In other words, when the two point-scatterers are spaced too closely, the assistance from Burg's BWE in the IBSR iteration is still achievable, but not when strong noise is involved. In this sense, the SR power of IBSR is limited by Laplace parameter estimation. On the other hand, this phenomenon also indicates that if some operations are capable of improving the accuracy of statistical estimation, the performance of IBSR can be further enhanced in some degree. This should be accounted for in further study.

\section{B. Real data set description and evaluation metrics}

In the following, real ground-based data is used for a performance analysis of BSR and IBSR for SR. Real data experimental comparisons of the Burg's BWE, CS, ICS, BSR, and IBSR are given. The performance analysis in the following is carried out by considering mainly two factors: the pulse amount and the noise interference.

The data set of a Yak-42 airplane is recorded by a Cband $(5.52 \mathrm{GHz})$ ISAR experimental system. The system transmits a $400-\mathrm{MHz}$ linear modulated chirp signal with $25.6-\mu$ s pulse duration, resulting in a range resolution of $0.375 \mathrm{~m}$. The received signal is de-chirped and I/Q sampled. In this data set, the pulse repetition frequency is $100 \mathrm{~Hz}$, i.e., 256 pulses within dwell time $[-1.28,1.28]$ (s) are utilized. Motion compensation, including range alignment and autofocus processing, is performed to remove the translational motion in advance. The aligned profiles are shown in Fig. 5a. The RD image generated 


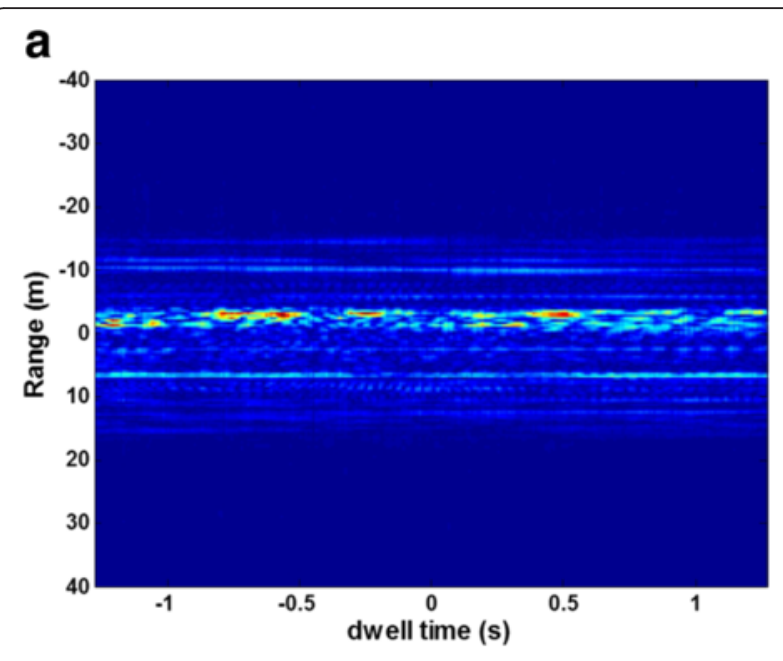

b



Fig. 5 Data set and RD image with 256 pulses. a Aligned range profiles. b RD image

by all 256 pulses is shown in Fig. $5 \mathrm{~b}$ for evaluating the experimental results. In the following experiments, SNR is defined as the energy ratio between the original data set and the added noise.

To provide a quantitative evaluation for the following SR imaging experiments, we consider some metrics that are directly relevant to the performance of automatic target recognition (ATR). The first metric is the coherence between the reconstructed SR image and the reference image in Fig. 5b, which represents the comparability of these two. In general, high coherence ensures high recognition probability in template-based classifiers [61]. The coherence is defined as follows.

$$
\text { Coherence }=\frac{\langle\mathbf{A} \odot \hat{\mathbf{A}}\rangle}{\|\mathbf{A}\|_{F} \cdot\|\hat{\mathbf{A}}\|_{F}}
$$

where $\mathbf{A}$ and $\hat{\mathbf{A}}=\left[\hat{a}_{n m}\right]_{\bar{N} \times M}$ denote the original and reconstructed SR image, respectively, $\odot$ denotes the
Hadamard product, and $<\cdot>$, the operator for summing up all components of a matrix. Notably, the coherence is defined with respect to the magnitude. Since the phases of the image are random, they usually provide few contributions to feature extraction for ATR [62]. Therefore, we calculate the coherence only with the magnitude of image in (36).

Another evaluation metric is the target-to-background ratio (TBR). By applying the adaptive watershed segmentation method [63] on the RD image to separate the target and the background regions and then counting the target energy (within the target region) and noise energy, and the TBR definition is given by

$$
\mathrm{TBR}=10 \cdot \log 10\left(\frac{\sum_{(n, m) \in T}\left|\hat{a}_{n m}\right|^{2}}{\sum_{(n, m) \in B}\left|\hat{a}_{n m}\right|^{2}}\right)
$$

where $T$ and $B$ are the target and background region, respectively, shown in Fig. 6. In our special case of imaging with limited noisy measurements, the main disadvantage of approaches based on parametric modeling is the introduction of false scattering centers. This could have a negative effect on the description of the configuration and features of the target. Clearly, TBR effectively considers the false points and energy leakage of target support. It is also relevant to the SNR of the reconstruction. Moreover, it can measure target preservation and noise suppression with the help of the predetermined target region. We define the noise energy as

$$
\text { Noise }=\sum_{(n, m) \in B}\left|\hat{a}_{n m}\right|^{2}
$$

The false points and noise in the background of the reconstructed image could be treated as scatters in ATR,

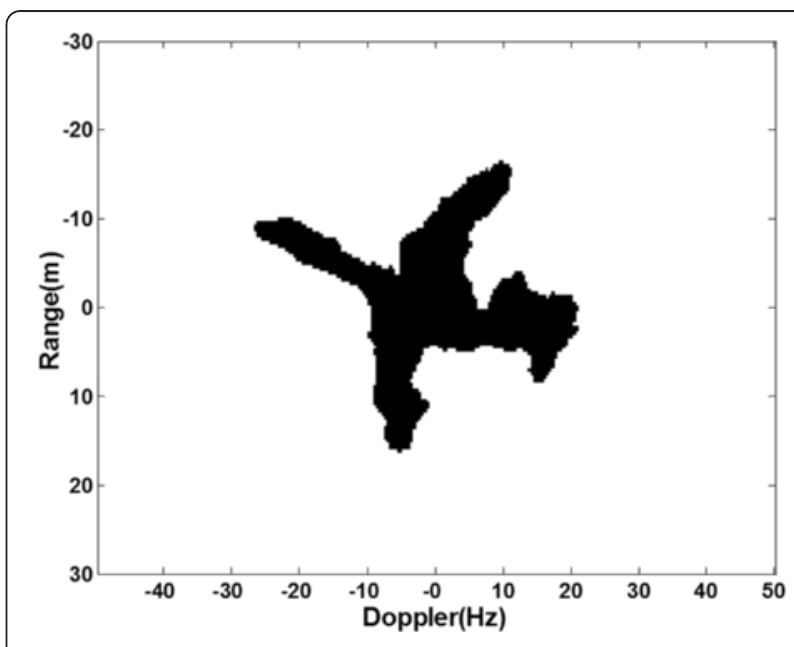

Fig. 6 Target region 
potentially leading to performance degradation. Both the TBR and noise energy metrics should be significant for feature-based ATR processing. We repeat the SR reconstruction 100 times with different noise in the following experiments to analyze coherence, TBR, and noise energy. The mean values of coherence, TBR, and noise energy from different SR approaches have been exploited to evaluate the experimental results in the following.

\section{Performance versus pulses amount}

To study how the sample amount affects the recovery performance, we compare the reconstructed results of BSR, IBSR, and current SR algorithms by varying the sample number. With different amount of pulses and constant SNR (10 dB), the SR images are generated with 256 Doppler bins. The pulse amount decreases from 128 to 16. In Fig. 7, we give typical images with four different pulse numbers $(128,64,32$, and 16), corresponding to 2 , 4, 8, and 16 times SR. By this, we investigate the performance of the five approaches with specific pulse numbers. In all processing of BSR and IBSR, CFAR is set to $10-4$ in the initialization step, the threshold for the Quasi-Newton solver is $\rho=10^{-3}$, and the terminating tolerance for CGA is $\delta_{\mathrm{CG}}=10^{-5}$. Typical experimental results are illuminated in Fig. 7. In Fig. 7, the first row presents the aperture patterns with different pulse numbers, the second row gives RD images after Burg's BWE, the third and fourth rows contain CS and ICS images, respectively, and the fifth and the bottom row show BSR and IBSR images, respectively.

From the comparisons, it is clear that ICS and IBSR perform better in scattering center extraction and noise suppression. In addition, IBSR imaging is more stable than BSR with a small number of pulses, as it generates dense and clear images with significant information and energy preserved to some extent. In order to characterize the performance quantitatively, the curves for the mentioned metrics with respective to the pulse amount are given in Fig. 8. Figure 8a provides the coherence between the recovered SR images and the reference image. Figure 8b, c shows the TBR and noise energy, respectively. It is worth noting that the performance of the IBSR and ICS performs well even under low SNR






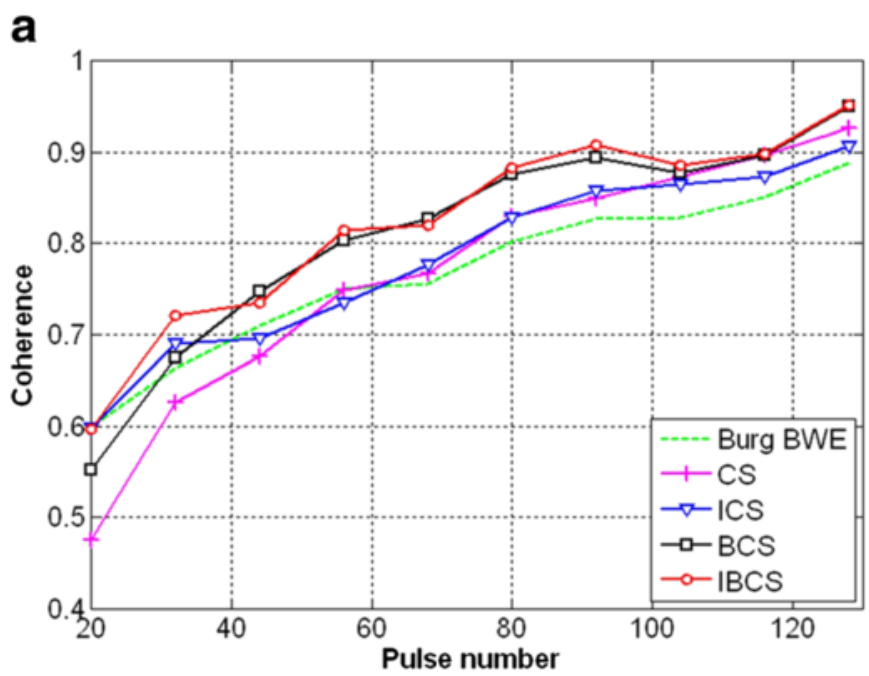

b

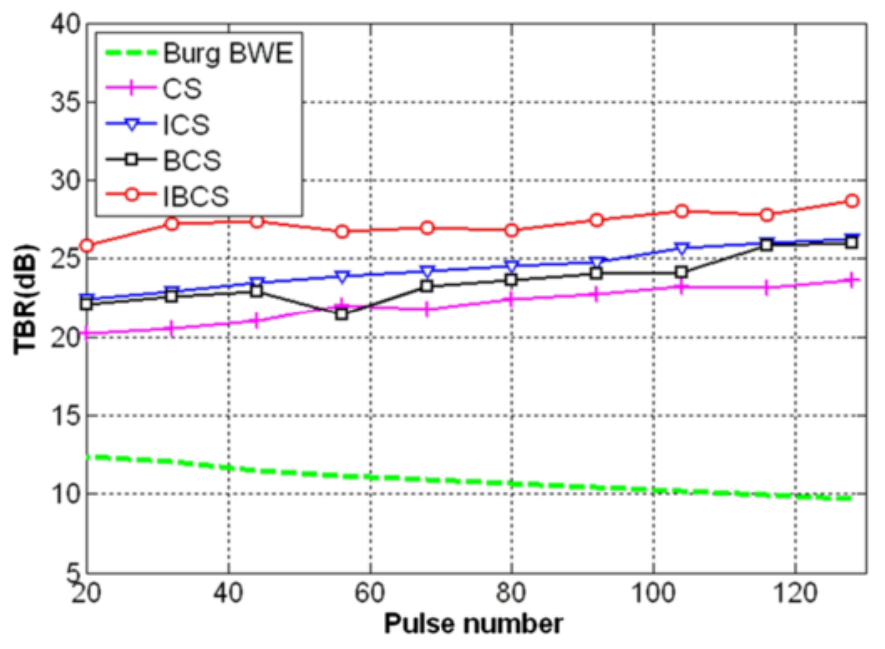

\section{C}



Fig. 8 Performance with different pulse numbers. a Coherence. b TBR. c Noise energy 
conditions. They provide higher coherence, TBR, and lower noise energy than the others. Figure 8a shows that the coherence corresponding to all algorithms increases with the increase of pulse amount. However, the sparsity-driven approaches can provide higher coherence than Burg's BWE even in the case of very limited pulses. Burg's BWE also presents good coherence performance, which lies in the fact that in Burg's BWE, the retention of primary data and phase coherency of the extrapolated signal are useful. In Fig. 8b, we note that Burg's BWE provides the lowest TBR because of its poor de-noising. CS, ICS, BSR, and IBSR give very high TBRs in a stable way. In particular, ICS and IBSR present TBR up to $25 \mathrm{~dB}$, indicating that they suppress the majority of noise while preserving target energy optimally, which is also clear in Fig. 8c. The metrics show that we achieve better performance by imposing proper statistics on the target and the nonidentical Laplace distribution is suitable to represent the sparsity of the image.

\section{Performance versus SNR}

The aim of this experiment is to analyze the influence of SNR on the performance of the approaches. To compare the noise tolerance of the four methods, we use 64 pulses corresponding to the dwell time [-0.32, 0.32] (s). As the original data set has high SNR after range compression, we generate experimental data with different SNRs $(15,10,5$, and $0 \mathrm{~dB})$ by adding complex-valued white Gaussian noise into the data. Then, four times aperture extrapolation is carried out with Burg's BWE, and a hamming windowed Fourier transform is then applied to yield a SR image with 256 Doppler bins. Correspondingly, SR images with the same Doppler bins obtained by CS and ICS are constructed with precise noise level. For BSR and IBSR implemented by the stage-by-stage flows, we need three iterations to yield 256-Doppler-bin images with 64 pulses. In Fig. 9, we give the resulting images with different SNRs. The recovered images via Burg's BWE, CS, ICS, BSR, and IBSR are shown from the first row to the fifth in Fig. 9, respectively. By comparing the results, we note that all the approaches provide good performance in the case of

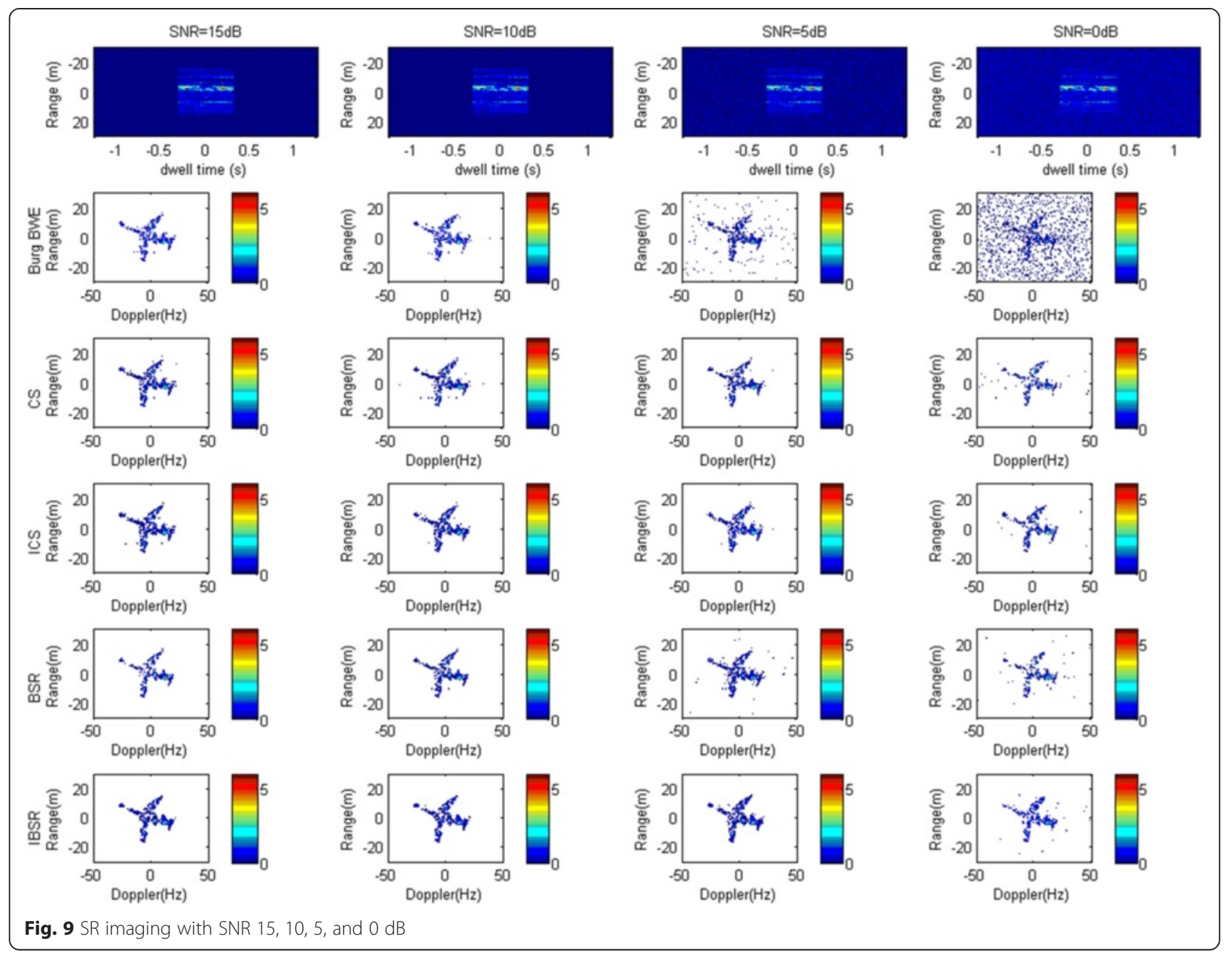


high SNR (15 and $10 \mathrm{~dB}$ ) and the images are formed in a clean and dense way. Nevertheless, artifacts increase apparently, and some scattering centers are missing when we decrease SNR in Burg's BWE images. The sparsity-driven methods are capable of providing good performance under strong noise. Distinctively, ICS and IBSR usually generate images with much fewer artificial background points and denser target images due to the weighting processing. Putting it quantitatively, the metric curves with respective to the SNR are given in Fig. 10. Figure 10a provides the coherence between the recovered SR images and the reference image. Figure 10b, c shows the TBR and noise energy, respectively. In Fig. 10a, we note that all algorithms provide stable coherence with varied SNRs. However, BSR provides comparable coherence to CS. ICS and IBSR give the highest coherence. In Fig. 10b, we note that TBR of all approaches decreases proportionally to the increase of noise energy. However, Burg's BWE generates the lowest TBR due to the lack of noise suppression. All sparsitydriven approaches perform much more stably, yielding high TBR up to $20 \mathrm{~dB}$ regardless of the decreasing SNR. In the aspect of TBR, IBSR is the best. In Fig. 10c, the noise energy of SR images obtained by the sparsitydriven approaches is distinctive from Burg's BWE. It is very low and almost constant with the decrease of SNR. Both the last and current experimental results show that sparsity-driven methods are optimal in SR imaging under noise conditions. In particular, ICS and IBSR can overcome strong noise and generate high-quality SR images effectively.

\section{Conclusions}

In this paper, we present SR imaging algorithms based on BCS. By combining the statistics estimation and CS, they are implemented by solving optimizations with $l_{1}$-norm optimization, derived from the MAP estimations. The sparsity level of the reconstructed image is determined by extracting the statistics from data stage by stage. Both BSR and IBSR are robust to strong noise and can reconstruct an image while suppressing strong noise. Based on the fact that the scattered field usually has distinctive spatial configuration, the nonidentical Laplace distribution is introduced in IBSR to discriminate prominent scattering centers from weak ones and noise in the $l_{1}$ penalty term. The nonidentical Laplace distribution promotes performance in terms of noise tolerance and limited pulses. The contribution of this paper illuminates the possibility of substantially improving the performance of SR imaging by leveraging statistical models coinciding with the image optimally. Surely, there are other novel statistics models that can also be used to represent the sparsity and local feature of radar image ideally.
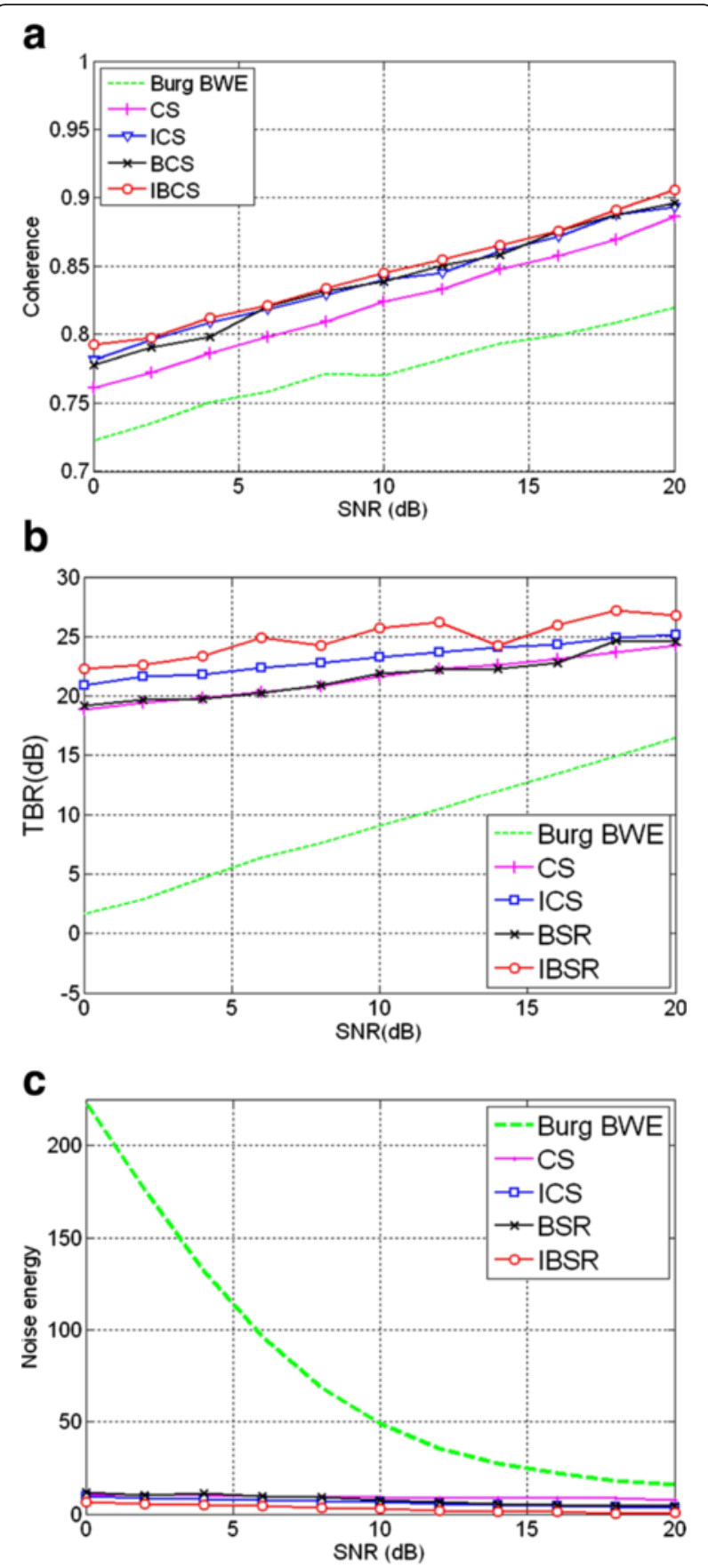

Fig. 10 Reconstruction metrics with different SNRs. a Coherence. b Target-to-background ratio. c Noise energy

Recent studies show that Gaussian mixture models [64] are optimal candidates to describe the structural dependence and sparsity of a target. This will be surveyed in future work. The improved statistical compressive sensing would be also beneficial for multiantenna setting for SAR [65] and ISAR imaging for maneuvering targets $[66,67]$. 


\section{Acknowledgements}

The authors thank the anonymous reviewers for their valuable comments. This work was supported by the National Natural Science Foundation of China (No. 61301280 and 61301293).

\section{Competing interests}

The authors declare that they have no competing interests.

\section{Author details}

'National Lab of Radar Signal Processing and the Collaborative Innovation Center of Information Sensing and Understanding, Xidian University, Xi'an 710071, People's Republic of China. ${ }^{2}$ School of Mathematical and Statistical Sciences, University of Texas-Rio Grande Valley, Edinburg TX 78539, USA.

Received: 29 April 2016 Accepted: 7 July 2016

Published online: 18 July 2016

\section{References}

1. Q Zhang, Y-Q Jin, Aspects of radar imaging using frequency-stepped chirp signals. EURASIP J Appl Signal Process 2006, 43-43 (2006). Available: http:// dx.doi.org/10.1155/ASP/2006/85823

2. SL Borison, SB Bowling, KM Cuomo, Super-resolution methods for wideband radar. Lincoln Lab J 5, 441-61 (1992)

3. JL Walker, Range-doppler imaging of rotating objects. Aerospace and Electronic Systems, IEEE Transactions on AES-16(1), 23-52 (1980)

4. SH, Image recovery: theory and practice (Academic, Orlando, 1987)

5. J Gudnason, J Cui, M Brookes, Hrr automatic target recognition from superresolution scattering center features. Aerospace and Electronic Systems, IEEE Transactions on 45(4), 1512-24 (2009)

6. LM Novak, G Owirka, AL Weaver, Automatic target recognition using enhanced resolution SAR data. Aerospace and Electronic Systems, IEEE Transactions on 35(1), 157-75 (1999)

7. H-J Li, N Farhat, Y Shen, A new iterative algorithm for extrapolation of data available in multiple restricted regions with application to radar imaging. Antennas and Propagation, IEEE Transactions on 35(5), $581-8$ (1987)

8. K Suwa, M Iwamoto, A two-dimensional bandwidth extrapolation technique for polarimetric synthetic aperture radar images. Geoscience and Remote Sensing, IEEE Transactions on 45(1), 45-54 (2007)

9. I Gupta, High-resolution radar imaging using 2-D linear prediction. Antennas and Propagation, IEEE Transactions on 42(1), 31-7 (1994)

10. TG Moore, BW Zuerndorfer, EC Burt, Enhanced imagery using spectralestimation-based techniques. Lincoln Lab J 10(no. 2), (1997)

11. RO Lane, KD Copsey, AR Webb, A Bayesian approach to simultaneous autofocus and superresolution (2004), pp. 133-42. Available: +http://dx.doi. org/10.1117/12.541504

12. A Mohammad-Djafari, G Demoment, Maximum entropy Fourier synthesis with application to diffraction tomography. Appl Optics 26(9), 1745-54 (1987)

13. J Li, P Stoica, Efficient mixed-spectrum estimation with applications to target feature extraction. Signal Processing. IEEE Transactions on 44(2) 281-95 (1996)

14. Z Bi, J Li, Z-S Liu, Super resolution SAR imaging via parametric spectral estimation methods. Aerospace and Electronic Systems, IEEE Transactions on 35(1), 267-81 (1999)

15. A Lazarov, Iterative MMSE method and recurrent Kalman procedure for ISAR image reconstruction. Aerospace and Electronic Systems, IEEE Transactions on $37(4), 1432-41$ (2001)

16. Z-S Liu, R Wu, J Li, Complex ISAR imaging of maneuvering targets via the capon estimator. Signal Processing, IEEE Transactions on 47(5), 1262-71 (1999)

17. M Palsetia, J Li, Using apes for interferometric SAR imaging. Image Processing, IEEE Transactions on 7(9), 1340-53 (1998)

18. J Li, P Stoica, An adaptive filtering approach to spectral estimation and SAR imaging. Signal Processing, IEEE Transactions on 44(6), 1469-84 (1996)

19. H Stankwitz, RJ Dallaire, J Fienup, Nonlinear apodization for sidelobe control in SAR imagery. Aerospace and Electronic Systems, IEEE Transactions on 31(1), 267-79 (1995)

20. $X X u, R$ Narayanan, Enhanced resolution in SAR/ISAR imaging using iterative sidelobe apodization. Image Processing, IEEE Transactions on 14(4), 537-47 (2005)

21. J Tsao, B Steinberg, Reduction of sidelobe and speckle artifacts in microwave imaging: the clean technique. Antennas and Propagation, IEEE Transactions on 36(4), 543-56 (1988)
22. R Bose, A Freedman, B Steinberg, Sequence clean: a modified deconvolution technique for microwave images of contiguous targets. Aerospace and Electronic Systems, IEEE Transactions on 38(1), 89-97 (2002)

23. D Donoho, Compressed sensing. Information Theory, IEEE Transactions on 52(4), 1289-306 (2006)

24. E Candes, T Tao, Near-optimal signal recovery from random projections: universal encoding strategies? Information Theory, IEEE Transactions on 52(12), 5406-25 (2006)

25. E Candes, M Wakin, An introduction to compressive sampling. Signal Processing Magazine, IEEE 25(2), 21-30 (2008)

26. KCetin, WC Karl, AS Willsky, Feature-preserving regularization method for complex-valued inverse problems with application to coherent imaging. Opt Eng 45(no. 1), 017 003-017 003-11 (2006). Available: +http://dx.doi.org/ $10.1117 / 1.2150368$

27. M Cetin, W Karl, Feature-enhanced synthetic aperture radar image formation based on nonquadratic regularization. Image Processing, IEEE Transactions on 10(4), 623-31 (2001)

28. G Zweig, Super-resolution Fourier transforms by optimisation, and ISAR imaging. Radar, Sonar and Navigation, IEE Proceedings 150(no. 4)، 247-52 (2003)

29. L Zhang, M Xing, C-W Qiu, J Li, Z Bao, Achieving higher resolution ISAR imaging with limited pulses via compressed sampling. Geoscience and Remote Sensing Letters, IEEE 6(3), 567-71 (2009)

30. XX Zhu, R Bamler, Tomographic SAR inversion by 11 norm regularization - the compressive sensing approach. IEEE Trans Geosci Remote Sens 48(10), 3839-46 (2010)

31. A Budillon, A Evangelista, G Schirinzi, Three-dimensional SAR focusing from multipass signals using compressive sampling. Geoscience and Remote Sensing, IEEE Transactions on 49(1), 488-99 (2011)

32. XX Zhu, R Bamler, Super-resolution power and robustness of compressive sensing for spectral estimation with application to spaceborne tomographic SAR. Geoscience and Remote Sensing, IEEE Transactions on 50(1), 247-58 (2012)

33. S Ji, Y Xue, L Carin, Bayesian compressive sensing. Signal Processing, IEEE Transactions on 56(6), 2346-56 (2008)

34. H Rohling, Radar CFAR thresholding in clutter and multiple target situations. Aerospace and Electronic Systems, IEEE Transactions on AES-19(4), 608-21 (1983)

35. Y Wang, $\mathrm{H}$ Ling, $V$ Chen, ISAR motion compensation via adaptive joint timefrequency technique. Aerospace and Electronic Systems, IEEE Transactions on 34(2), 670-7 (1998)

36. W Ye, TS Yeo, Z Bao, Weighted least-squares estimation of phase errors for SAR/ISAR autofocus. Geoscience and Remote Sensing, IEEE Transactions on 37(5), 2487-94 (1999)

37. J Wang, D Kasilingam, Global range alignment for ISAR. Aerospace and Electronic Systems, IEEE Transactions on 39(1), 351-7 (2003)

38. D Zhu, L Wang, Y Yu, Q Tao, Z Zhu, Robust ISAR range alignment via minimizing the entropy of the average range profile. Geoscience and Remote Sensing Letters, IEEE 6(2), 204-8 (2009)

39. TJ Kragh, Monotonic iterative algorithm for minimum-entropy autofocus, in Adaptive Sensor Array Processing (ASAP) Workshop],(June 2006) (2006)

40. Q Zhang, T-S Yeo, G Du, S Zhang, Estimation of three-dimensional motion parameters in interferometric ISAR imaging. Geoscience and Remote Sensing, IEEE Transactions on 42(2), 292-300 (2004)

41. F Berizzi, G Corsini, Autofocusing of inverse synthetic aperture radar images using contrast optimization. Aerospace and Electronic Systems, IEEE Transactions on 32(3), 1185-91 (1996)

42. MAT Figueiredo, Adaptive sparseness for supervised learning. Pattern Analysis and Machine Intelligence, IEEE Transactions on 25(9), 1150-9 (2003)

43. E Vera, L Mancera, S Babacan, R Molina, A Katsaggelos, Bayesian compressive sensing of wavelet coefficients using multiscale laplacian priors, in Statistical Signal Processing, 2009. SSP'09 (IEEE/SP 15th Workshop on, 2009), pp. 229-32

44. NL Pedersen, D Shutin, CN Manchón, BH Fleury, Sparse estimation using Bayesian hierarchical prior modeling for real and complex models (, 2011) arXiv preprint arXiv:1108.4324

45. DF Andrews, CL Mallows, Scale mixtures of normal distributions. J R Stat Soc Ser B Methodol 36(no. 1), 99-102 (1974). Available: http://www.jstor.org/ stable/2984774

46. S Yu, AS Khwaja, J Ma, Compressed sensing of complex-valued data. Signal Process 92(no. 2), 357-62 (2012). http://www.sciencedirect.com/science/ article/pii/S0165168411002568 
47. S Chen, D Donoho, M Saunders, Atomic decomposition by basis pursuit. SIAM J Sci Comput 20(no. 1), 33-61 (1998). http://epubs.siam.org/doi/abs/ $10.1137 /$ S1064827596304010

48. C Zhu, Stable recovery of sparse signals via regularized minimization. Information Theory, IEEE Transactions on 54(7), 3364-7 (2008)

49. E van den Berg, MP Friedlander, In pursuit of a root (2007). preprint

50. EJ Candes, MB Wakin, SP Boyd, Enhancing sparsity by reweighted I 1 minimization. J Fourier Anal Appl 14(5-6), 877-905 (2008)

51. M Richards, Fundamentals of radar signal processing, ser. Professional Engineering (Mcgraw-hill, 2005). Available: http://books.google.com/ books?id=VOvsJ7G1oDEC

52. G Davidson, Radar toolbox (2003). cited August 2013. [Online]. Available: http://www.radarworks.com/software.htm

53. P-R Wu, A criterion for radar resolution enhancement with burg algorithm. Aerospace and Electronic Systems, IEEE Transactions on 31(3), 897-915 (1995)

54. JF Sturm, Using SeDuMi 1.02, a Matlab toolbox for optimization over symmetric cones. Optimization methods and software 11(1-4), 625-53 (1999)

55. VCSDL Donoho, I Driori, Y Tsaig, Sparselab (2007). Available: http://sparselab. stanford.edu/

56. SBM Grant, Y Ye, cvX: Matlab software for disciplined convex programming (" 2013). Available: http://www.stanford.edu/ boyd/cvx/

57. Y Zhang, Yall1 (2011). Available: http://www.caam.rice.edu/ optimization/ L1/YALL1/

58. CR Vogel, ME Oman, Fast, robust total variation-based reconstruction of noisy, blurred images. Image Processing, IEEE Transactions on 7(6), 813-24 (1998)

59. O Axelsson, Iterative solution methods (Cambridge University Press, 1996)

60. SP Boyd, L Vandenberghe, Convex optimization (Cambridge Univ Press, 2004)

61. L Zhang, M Xing, C-W Qiu, J Li, J Sheng, Y Li, Z Bao, Resolution enhancement for inversed synthetic aperture radar imaging under low SNR via improved compressive sensing. Geoscience and Remote Sensing, IEEE Transactions on 48(10), 3824-38 (2010)

62. M Cetin, WC Karl, D Castanon, Feature enhancement and ATR performance using nonquadratic optimization-based SAR imaging. Aerospace and Electronic Systems, IEEE Transactions on 39(4), 1375-95 (2003)

63. MN Saidi, MN Saidi, A Toumi, B Hoeltzener, A Khenchaf, D Aboutajdine, ISAR data dynamics: target shapes features extraction for the design of ISAR retrieval system, in Synthetic Aperture Radar (EUSAR) (European Conference on, 2008), pp. 1-4

64. G Yu, G Sapiro, Statistical compressed sensing of gaussian mixture models. Signal Processing, IEEE Transactions on 59(12), 5842-58 (2011)

65. J Lopez, Z Qiao, Array geometries, signal type, and sampling conditions for the application of compressed sensing in MIMO radar, in SPIE Defense, Security, and Sensing (International Society for Optics and Photonics, 2013), p. 871. 702-871702

66. W Rao, G Li, X Wang, X-G Xia, Adaptive sparse recovery by parametric weighted I1 minimization for ISAR imaging of uniformly rotating targets. IEEE J Selected Topics in Applied Earth Observations and Remote Sensing 6(2), 942-52 (2012)

67. L Zhang, I Duan, Z Qiao, M Xing, Z Bao, Phase adjustment and isar imaging of maneuvering targets with sparse apertures. Aerospace and Electronic Systems, IEEE Transactions on 50(3), 1955-73 (2014)

\section{Submit your manuscript to a SpringerOpen ${ }^{\circ}$ journal and benefit from:}

- Convenient online submission

- Rigorous peer review

- Immediate publication on acceptance

- Open access: articles freely available online

- High visibility within the field

- Retaining the copyright to your article 